\title{
Positive Definiteness of Multivariate Densities Based on Hermite Polynomials
}

\author{
Javier Perote $^{1}$ and Esther B. del Brío ${ }^{2}$
}

${ }^{1}$ Corresponding author: Universidad Rey Juan Carlos. Dept. de Economía Aplicada II y Fundamentos del Análisis Económico. Fac. de Ciencias Jurídicas y Sociales. Campus de Vicálvaro. P ${ }^{o}$ de los Artilleros s/n. 28032 Madrid (Spain). Tel: +34 91488 7684. E-mail: perote@fcjs.urjc.es

${ }^{2}$ Universidad de Salamanca. Dept. de Administración y Economía de la Empresa. Edificio F.E.S.. Campus Miguel de Unamuno. 37007. Salamanca (Spain). Tel: +34 923 294640. E-mail: ebrio@usal.es 


\begin{abstract}
This paper develops both univariate and multivariate distributions based on Gram-Charlier and Edgeworth expansions, attempting to ensure non negativity by exploiting the orthogonal properties of the Hermite polynomials. The article motivates the problems underlying some specifications (in particular those involving other conditional moments beyond the variance) and provides empirical examples comparing the performance of these positive definite densities to the univariate and multivariate versions of the so-called Edgeworth-Sargan distribution when fitting stock market indices. The fitted densities perform similarly and thus the use of the positive versions depends on other econometric considerations rather than accuracy.
\end{abstract}

Key words: Gram-Charlier and Edgeworth expansions, conditional heterosckedasticity and kurtosis, financial data.

JEL classification: C16, G1. 


\section{INTRODUCTION}

The Gram-Charlier and the Edgeworth expansions were established in the end of the 19th century and the beginning of the 20th century by Edgeworth (1896), Charlier (1905) and Edgeworth (1907). Since then, these expansions have been used in many fields from mathematics or statistics to physics, but it was Sargan in the seventies who brought these expansions into econometrics - Sargan (1975) and Sargan (1976). More recently the densities based on these expansions have also been investigated - Nishiyama and Robinson (2000), Velasco and Robinson (2001) and Nabeya (2001) - and have been introduced in finance to capture the asymmetric and leptokurtic behaviour of high frequency financial data - see Mauleón (1997), Mauleón and Perote (2000) or Verhoeven and McAller (2004) as recent examples. Even more, these densities have also been used to price options - Jarrow and Rudd (1982), Corrado and Su (1996) and Rubinstein (1998) - or to improve Value at Risk (VaR) measures - see Perote and Del Brío (2003) or Christoffersen and Gonçalves (2003).

All these articles reveal densities based on Hermite polynomials as accurate and general specifications that capture skewness and kurtosis of most high frequency data - in particular, Mauleón and Perote (2000) proved that these densities are capable of capturing financial data behavior even better than other non-normal distributions such as the Student's t. Nevertheless, these distributions based on Hermite expanisons are not always positive definite. This problem was already higlighted by Barton and Dennis (1952) and Draper and Tierny (1972) and also in more recent papers such as Joundeau and Rockinger (2001). Different solutions to this problem in 
univariate contexts were introduced by authors such as Gallant and Nychka (1987) and Gallant and Tauchen (1989). More recently, León et al. (2004) have proposed some variations to the conditional variance and kurtosis model defined by Harvey and Siddique (1999) in an attempt to ensure positive definiteness.

Moreover, the analysis of multivariate financial data have been performed by using many multivariate distributions and also multivariate GARCH structures - see Bauwens et al. (2005) for a recent survey on multivariate GARCH models. Nevertheless, as far as we know, the Gram-Charlier and Edgeworth expansions have scarcely been introduced to define multivariate densities. Actually, Perote (2004), shows the first attempt to develop a multivariate density based on Gaussian expansions by defining the multivariate Edgeworth-Sargan distribution. The resulting density, called the Multivariate Edgeworth-Sargan distribution, was proved to fit high frequency financial data even better than Prucha and Kelejian's (1984) multivariate Student's t by means of its general and flexible representation. Even more, this multivariate density was used in Perote and Del Brío (2003) to improve Value-at-Risk measures of non-normal portfolios. Nevertheless, despite its utility, this multivariate distribution also presents the shortcoming of not being always positive definite. This is the main stress of our article, which sheds light on this issue by providing different positive definite specifications of this kind of densities but preserving the clear advantages of their former non-positive counterparts.

The remaining of the article is structured as follows: Section 2 is devoted to specifying univariate densities based on Hermite polynomials focusing 
on different solutions to prevent negative values; Section 3 discusses the use of the different specifications, specially when conditional moments are considered; Section 4 introduces several types of multivariate positive definite densities; Section 5 provides empirical estimates for some of the univariate and multivariate densities defined throughout the paper, and the last section (Section 6) gathers the main conclusions.

\section{FINDING POSITIVE DENSITIES BASED ON HERMITE POLYNOMIALS}

The Gram-Charlier and Edgeworth series have been used to define general density specifications whose moments are directly related to some parameters $\left(d_{s}\right)$ weighting the so called Hermite polynomials. These polynomials, $H_{s}(x)$, are defined in terms of the derivatives of the normal density, $g(x)$, as expressed in Eq. (1).

$$
\frac{d^{s} g(x)}{d x^{s}}=(-1)^{s} H_{s}(x) g(x)
$$

or alternatively, they can be worked out by using the following closed form - see Kendall and Stuart (1977):

$$
H_{s}(x)=\left\{\begin{array}{l}
\sum_{i=0}^{s / 2}(-1)^{i} x^{s-2 i} \frac{s !}{2^{i} i !(s-2 i) !} \text { if } s \text { is even } \\
\sum_{i=0}^{s-1) / 2}(-1)^{i} x^{s-2 i} \frac{s !}{2^{i} i !(s-2 i) !} \text { if } s \text { is odd }
\end{array}\right.
$$


These polynomials satisfy, among others, interesting orthogonality properties. In particular it is proved that $\forall s, j=0,1,2, \ldots$ (see also Kendall and Stuart (1977) for further details)

$$
\begin{gathered}
\int H_{s}(x) H_{j}(x) g(x) d x=0 \text { if } s \neq j \text { and } \\
\int H_{s}(x) H_{j}(x) g(x) d x=s ! \text { if } s=j .
\end{gathered}
$$

Such orthogonality conditions guarantee that it is possible to define functions based on Hermite polynomials that integrate up to one, the simplest case being the Edgeworth-Sargan distribution used in some articles such as Mauleón and Perote (2000). This distribution is defined as the expansion of the Gaussian density truncated at the order $q$ - see Eq. (5).

$$
f_{1}(x)=g(x)\left[1+\sum_{s=1}^{q} d_{s} H_{s}(x)\right]
$$

This distribution is easily proved to integrate one - as a direct application of Eq. (3) to the Hermite polynomial $H_{0}(x)=1$ - but it is not strictly a density since this function is not positive for all the values of the parameter space. However, Mauleón and Perote (2000) emphasised the fact that accurate optimisation procedures usually converge to parameter values guaranteeing the positivity of the density. Other authors, e.g. Joundeau and Rockinger (2001), found out parameter restrictions to achieve non negativity. Nevertheless, there exist other more fruitful approaches to ensure positivity 
such as Gallant and Nychka (1987) and Gallant and Tauchen (1989) who introduced straightforward solutions to this problem that lied in defining the density in terms of the squared weighted sum of Hermite polynomials as shown below.

$$
f_{2}(x)=g(x)\left[d_{0}+\left(\sum_{s=1}^{q} d_{s} H_{s}(x)\right)^{2}\right]
$$

where

$$
d_{0}=1-\int g(x)\left(\sum_{s=1}^{q} d_{s} H_{s}(x)\right)^{2} d x=1-\sum_{s=1}^{q} d_{s}^{2} s ! .
$$

Nevertheless, this distribution requires additional constrains (such as $\left.d_{0}>0\right)$ to ensure non negativity - see Mauleón (2003) - and, consequently, we explore other possibilities that in general do not need further restrictions. In particular we propose different specifications - see Eqs. (8), (9) and (10) - exploiting the orthogonality properties of the Hermite polynomials - Eqs. (3) and (4).Particularly, the density displayed in Eq. (10) was called Positive Edgeworth-Sargan by Niguez and Perote (2004).

$$
\begin{gathered}
f_{3}(x)=g(x)\left[1+\sum_{s=1}^{q} d_{s} H_{s}(x)\right]^{2} \frac{1}{k} \\
f_{4}(x)=g(x)\left[1+\left(\sum_{s=1}^{q} d_{s} H_{s}(x)\right)^{2}\right] \frac{1}{k} \\
f_{5}(x)=g(x)\left[1+\sum_{s=1}^{q} d_{s}^{2} H_{s}(x)^{2}\right] \frac{1}{k}
\end{gathered}
$$


For all of these three densities it is proved that the constant $k$, which make them integrate up to one, is that of the Eq. (11) - see Proof 1 in the Appendix.

$$
\begin{aligned}
k & =\int g(x)\left[1+\sum_{s=1}^{q} d_{s} H_{s}(x)\right]^{2} d x=\int g(x)\left[1+\left(\sum_{s=1}^{q} d_{s} H_{s}(x)\right)^{2}\right] d x \\
& =\int g(x)\left[1+\sum_{s=1}^{q} d_{s}^{2} H_{s}(x)^{2}\right] d x=1+\sum_{s=1}^{q} d_{s}^{2} s !
\end{aligned}
$$

It is worth noticing, as well, that these three densities seem to be more appropriate than the one shown in Eq. (6) not only from a theoretical perspective but also from and empirical viewpoint, since the log-likelihood function is separable - see Eq. (12) - and involves logarithms of positive terms, thus simplifying the optimisation procedures.

$$
L n L=\ln [g(x)]+\ln \left[\left(1+\sum_{s=1}^{q} d_{s} H_{s}(x)\right)^{2}\right]-\ln \left[1+\sum_{s=1}^{q} d_{s}^{2} s !\right]
$$

\section{WHICH POSITIVE DENSITY TO CHOOSE?}

In the previous section we showed that there exist different positive density specifications based on Gram-Charlier and Edgeworth series expansions that can be used to fit the distribution underlying most high frequency data. Therefore, from an empirical perspective, we might wonder which specification seems to be more adequate. Unfortunately, there is no general answer to this question because the final choice depends on the econometric targets, the nature of the data and the remaining assumptions of the model. 
For example, if we focus on the in-sample performance and the presence of outliers is not an important shortcoming, the traditional Edgeworth-Sargan density shown in Eq. (5) might be the best option. On the other hand, if the objective is forecasting and potential outliers are likely to happen using densities that ensure positivity would be strongly recommended. Even more, the success on obtaining accurate estimates and the appropriate interpretation of the results lie in the relationship between the density moments and its parameters. For that reason, the in-depth analysis of these relations is the key to choose the more accurate specification.

In what follows we consider some examples to motivate the importance of analysing and interpreting the parameters of the proposed densities. Let us imagine that we are modelling the conditional density of a high frequency financial variable, $y_{t}$, which has a strong persistence of volatility clusters. For such a variable, it seems to be plausible the consideration of $\mathrm{ARCH}$ or GARCH structures - proposed by Engle (1982) and Bollerslev (1986) not only for capturing conditional variance, i.e $\sigma_{t}^{2}=E_{t-1}\left[\left(y_{t}-\mu_{t}\right)^{2}\right]$, but also to account for conditional kurtosis - Harvey and Siddique (1999) used a $\operatorname{GARCH}(1,1)$ to model skewness and León et al. (2004) attempted to measure kurtosis likewise -, i.e. $k_{t}^{4}=\frac{1}{\sigma_{t}^{4}} E_{t-1}\left[\left(y_{t}-\mu_{t}\right)^{4}\right],\left(\mu_{t}=E_{t-1}\left[y_{t}\right]\right.$ being the conditional mean of $y_{t}$ ). Thus the density of the standardised variable $\varepsilon_{t}=\frac{y_{t}-\mu_{t}}{\sigma_{t}}$ might be modelled according to $f_{i}(\cdot) \forall i=1,3,4-$ see Eqs. (5), (8) and (9). For the sake of simplicity, we concentrate on these three densities constrained to $d_{s}=0 \forall s \neq 4$, and hence the resulting densities are given in Eqs. (13), (14) and (15), respectively. 


$$
\begin{gathered}
f_{1}\left(\varepsilon_{t}\right)=g\left(\varepsilon_{t}\right)\left[1+d_{4 t}\left(\varepsilon_{t}^{4}-6 \varepsilon_{t}^{2}+3\right)\right] \\
f_{3}\left(\varepsilon_{t}\right)=\frac{1}{1+24 d_{4 t}^{2}} g\left(\varepsilon_{t}\right)\left[1+d_{4 t}\left(\varepsilon_{t}^{4}-6 \varepsilon_{t}^{2}+3\right)\right]^{2} \\
f_{4}\left(\varepsilon_{t}\right)=\frac{1}{1+24 d_{4 t}^{2}} g\left(\varepsilon_{t}\right)\left[1+d_{4 t}^{2}\left(\varepsilon_{t}^{4}-6 \varepsilon_{t}^{2}+3\right)^{2}\right]
\end{gathered}
$$

Note that, although in most distributions based on Edgeworth and GramCharlier expansions the odd moments depend on the odd parameters, it is easy to check that the densities proposed in (8) and (9) may also account for asymmetries even when all the odd parameters are constrained to zero. Nevertheless, the former three densities are all symmetric and their even moments are different functions of the density parameter $d_{4 t}$. In particular, the second and fourth moments of the densities (13), (14), and (15) are given in Eqs. (16), (17) and (18), respectively (see Proof 2 in the Appendix).

$$
\begin{gathered}
E_{t-1}\left[\varepsilon_{t}^{2}\right]=1 \text { and } E_{t-1}\left[\varepsilon_{t}^{4}\right]=3+24 d_{4 t} \\
E_{t-1}\left[\varepsilon_{t}^{2}\right]=\frac{1+216 d_{4 t}^{2}}{1+24 d_{4 t}^{2}} \text { and } E_{t-1}\left[\varepsilon_{t}^{4}\right]=\frac{3+2952 d_{4 t}^{2}+12 d_{4 t}}{1+24 d_{4 t}^{2}} \\
E_{t-1}\left[\varepsilon_{t}^{2}\right]=\frac{1+216 d_{4 t}^{2}}{1+24 d_{4 t}^{2}} \text { and } E_{t-1}\left[\varepsilon_{t}^{4}\right]=\frac{3+2952 d_{4 t}^{2}}{1+24 d_{4 t}^{2}}
\end{gathered}
$$


Note that in some cases, such as the densities (13) and (15), the relations given in (16) and (18) can be used to rewrite the densities in terms of the fourth moment of the variable, as is shown in (19) and (20), respectively.

$$
\begin{gathered}
f_{1}\left(\varepsilon_{t}\right)=g\left(\varepsilon_{t}\right)\left[1+\frac{E_{t-1}\left[\varepsilon_{t}^{4}\right]-3}{24}\left(\varepsilon_{t}^{4}-6 \varepsilon_{t}^{2}+3\right)\right] \\
f_{4}\left(\varepsilon_{t}\right)=\frac{123-E_{t-1}\left[\varepsilon_{t}^{4}\right]}{120} g\left(\epsilon_{t}\right)\left[1+\frac{E_{t-1}\left[\varepsilon_{t}^{4}\right]-3}{24\left(123-E_{t-1}\left[\varepsilon_{t}^{4}\right]\right)}\left(\varepsilon_{t}^{4}-6 \varepsilon_{t}^{2}+3\right)^{2}\right]
\end{gathered}
$$

Moreover, if conditional variance and kurtosis processes are considered, such as the $\operatorname{GARCH}(1,1)$ processes displayed in (21) and (22), the conditional densities of the variable $u_{t}=\sigma_{t} \varepsilon_{t}$ corresponding to the densities (19) and (20) will be given respectively by (23) and (24).

$$
\begin{aligned}
& \sigma_{t}^{2}=\alpha_{0}+\alpha_{1} u_{t-1}^{2}+\alpha_{2} \sigma_{t-1}^{2} \\
& k_{t}^{4}=\beta_{0}+\beta_{1}\left(\frac{u_{t-1}}{\sigma_{t-1}}\right)^{4}+\beta_{2} k_{t-1}^{4} \\
& f_{1}\left(u_{t}\right)=\frac{1}{\sigma_{t}} g\left(\frac{u_{t}}{\sigma_{t}}\right)\left[1+\frac{k_{t}^{4}-3}{24}\left[\left(\frac{u_{t}}{\sigma_{t}}\right)^{4}-6\left(\frac{u_{t}}{\sigma_{t}}\right)^{2}+3\right]\right] \\
& f_{4}\left(\varepsilon_{t}\right)=\frac{123-k_{t}^{4}}{120} \frac{1}{\sigma_{t}} g\left(\frac{u_{t}}{\sigma_{t}}\right)\left[1+\frac{k_{t}^{4}-3}{24\left(123-k_{t}^{4}\right)}\left[\left(\frac{u_{t}}{\sigma_{t}}\right)^{4}-6\left(\frac{u_{t}}{\sigma_{t}}\right)^{2}+3\right]^{2}\right]
\end{aligned}
$$


Nevertheless it is noteworthy the fact that the conditional kurtosis version of $f_{4}(\cdot)$, i.e. the equation $(24)$, is only positive for leptokurtic distributions such that $3<k_{t}^{4}<123$ and, despite such condition is held in most high frequency financial data, for some outliers positivity could be jeopardised. That means that there is a trade off between considering conditional moments beyond the variance and positive definiteness (this issue is also clear in asymmetric distributions based on Hermite polynomials). Therefore defining positive definite densities that account simultaneously for different conditional moments and that do not involve awkward restrictions is still an open question that deserves further research. In this sense the stationarity conditions of the GARCH processes introduced in these densities must also be carefully analysed, since the conditional variance of $u_{t}$ (hereafter, $\left.\sigma_{t}^{2+}\right)$, depends on $E_{t-1}\left[\varepsilon_{t}^{2}\right]$ as shown below:

$$
\sigma_{t}^{2+}=\alpha_{0}^{+}+\alpha_{1}^{+} u_{t-1}^{2}+\alpha_{2} \sigma_{t-1}^{2+}
$$

where $\alpha_{0}^{+}=\alpha_{0} E_{t-1}\left[\varepsilon_{t}^{2}\right]$ and $\alpha_{1}^{+}=\alpha_{1} E_{t-1}\left[\varepsilon_{t}^{2}\right]$. Even more, from (18) it follows that

$$
E_{t-1}\left[\varepsilon_{t}^{2}\right]=\frac{k_{t}^{4}+12}{15}
$$

(also observe that $E_{t-1}\left[\varepsilon_{t}^{2}\right]>1$, provided that $k_{t}^{4}>3$ ) and thus the stationarity condition $\alpha_{1}^{+}+\alpha_{2}<1$ involves additional constrains to the conditional kurtosis, such as 


$$
k_{t}^{4}<\frac{\left(1-\alpha_{2}\right) 15-12 \alpha_{1}}{\alpha_{1}} .
$$

All this discussion illustrates some alternatives to searching appropriate univariate densities based on Hermite polynomials and emphasising the fact that guaranteeing positive definiteness is often possible, but the final specification must involve a detailed study of the statistical properties of the density and should be guided by the nature of the data and the econometric assumptions.

\section{POSITIVE DEFINITE MULTIVARIATE DENSITIES}

The increasing correlation among financial markets highlights the need of studying the joint behaviour of high frequency financial variables by estimating the multivariate p.d.f. of groups of variables. For that purpose the Edgeworth and Gram-Charlier series can also be used and the type of densities shown in previous epigraphs can be straightforwardly extended to a multivariate framework. The simplest case is given in Perote (2004), which generalises the Edgeworth-Sargan density - see Eq. (5) - to a $n$-variate context. The resulting Multivariate Edgeworth-Sargan (hereafter, MES) provides a joint distribution whose marginal densities behave as univariate Edgeworth-Sargan, which not only facilitates the parameter interpretation 
but also simplifies the estimating procedures. In particular, for a vector $X^{\prime}=\left[\begin{array}{llll}x_{1} & x_{2} & \cdots & x_{n}\end{array}\right]$, this density is defined as

$$
F(X)=G(X)+\prod_{i=1}^{n} g\left(x_{i}\right) \sum_{i=1}^{n} \sum_{s=1}^{q} d_{s i} H_{s}\left(x_{i}\right)
$$

where $G(X)$ represents a multivariate normal distribution with zero mean and variance matrix $\Sigma$ - as shown in (29) - whose marginal densities are the univariate normals $g\left(x_{i}\right)$.

$$
G(X)=(2 \pi)^{-n / 2}\left|\Sigma^{-1 / 2}\right| \exp \left\{-\frac{1}{2} X^{\prime} \Sigma^{-1} X\right\}
$$

In order to consider a "standard" density, at the outset we assume that every variance is one and the correlation coefficients of $x_{i}$ and $x_{j}$ are $\rho_{i j}$ for all $i \neq j$. Therefore, all the moments of the distribution depend on the correlation coefficients and $d_{s i} \forall s=1,2, \ldots, q$ and $\forall i=1,2, \ldots, n$. For the sake of clarity the bivariate case of this distribution (for the variables $x_{t}$ and $y_{t}$ ) is given in equation (30). Without loss of generality, we expand every marginal Gaussian density to the same order $q$. Moreover $d_{s x}$ and $d_{s y}$ stand for the $s^{t h}$ order Hermite polynomial parameters corresponding to the variables $x_{t}$ and $y_{t}$ respectively. For this particular case and without loss of generality it can be easily proved that the marginal density for the variable $x_{t}$ is in fact distributed according to (5) (see Proof 3 in the Appendix).

$$
F\left(x_{t}, y_{t}\right)=G\left(x_{t}, y_{t}\right)+g\left(x_{t}\right) g\left(y_{t}\right)\left[\sum_{s=1}^{q} d_{s x} H_{s}\left(x_{t}\right)+\sum_{s=1}^{q} d_{s y} H_{s}\left(y_{t}\right)\right]
$$


This distribution has been found out to be very useful for capturing the joint density of financial variables, but on the other hand the estimation techniques for such density require a very accurate selection of initial values (based on the previous estimates for the marginal densities) so as to prevent processes from non converging due to potential negative values for the density (note that maximising the log-likelihood for a given sample is not compatible with parameter estimates approaching to zero, which would imply that the log likelihood tends to minus infinite). For that reason, in this paper we propose two possible variations of (28) to ensure that $F(X) \geq 0$ for all $X$, which we call the Multivariate Gram-Charlier I and the Multivariate GramCharlier II (hereafter MGCI and MGCII, respectively).The former, MGCI, implements the same density structure displayed in (8) into the MES,

$$
F_{I}(X)=\frac{1}{n+1} G(X)+\frac{1}{n+1} \prod_{i=1}^{n} g\left(x_{i}\right) \sum_{i=1}^{n} \frac{1}{k_{i}}\left[1+\sum_{s=1}^{q} d_{s i} H_{s}\left(x_{i}\right)\right]^{2}
$$

where

$$
k_{i}=\int g\left(x_{i}\right)\left[1+\sum_{s=1}^{q} d_{s i} H_{s}\left(x_{i}\right)\right]^{2} d x_{i}=1+\sum_{s=1}^{q} d_{s i}^{2} s !
$$

Particularly, the bivariate distribution for variables $x_{t}$ and $y_{t}$ can be written as follows:

$F_{I}\left(x_{t}, y_{t}\right)=\frac{1}{3} G\left(x_{t}, y_{t}\right)+\frac{1}{3} g\left(x_{t}\right) g\left(y_{t}\right)\left[\frac{1}{k_{x}}\left[1+\sum_{s=1}^{q} d_{s x} H_{s}\left(x_{t}\right)\right]^{2}+\frac{1}{k_{y}}\left[1+\sum_{s=1}^{q} d_{s y} H_{s}\left(y_{t}\right)\right]^{2}\right]$ 
where

$$
\begin{gathered}
k_{x}=\int g\left(x_{t}\right)\left[1+\sum_{s=1}^{q} d_{s i} H_{s}\left(x_{t}\right)\right]^{2} d x_{t}=1+\sum_{s=1}^{q} d_{s x}^{2} s ! \text { and } \\
k_{y}=\int g\left(y_{t}\right)\left[1+\sum_{s=1}^{q} d_{s i} H_{s}\left(y_{t}\right)\right]^{2} d y_{t}=1+\sum_{s=1}^{q} d_{s y}^{2} s !
\end{gathered}
$$

This distribution is indeed a density since it also integrates up to one and its marginal densities are also univariate density functions. For instance, the marginal density of $x_{t}$ is that of the Eq. (36) (see Proof 3 in the Appendix).

$$
f_{I}\left(x_{t}\right)=\frac{2}{3} g\left(x_{t}\right)+\frac{1}{3 k_{x}} g\left(x_{t}\right)\left[1+\sum_{s=1}^{q} d_{s x} H_{s}\left(x_{t}\right)\right]^{2}
$$

On the other hand, the MGCII is the multivariate case of the Eq. (9) and may be expressed as follows,

$$
F_{I I}(X)=\frac{1}{n+1} G(X)+\frac{1}{n+1} \prod_{i=1}^{n} g\left(x_{i}\right) \sum_{i=1}^{n} \frac{1}{c_{i}}\left[\sum_{s=1}^{q} d_{s i} H_{s}\left(x_{i}\right)\right]^{2}
$$

where

$$
c_{i}=\int g\left(x_{i}\right)\left[\sum_{s=1}^{q} d_{s i} H_{s}\left(x_{i}\right)\right]^{2} d x_{i}=\sum_{s=1}^{q} d_{s i}^{2} s !
$$

Note that as far as MGCII is defined the constants ensuring the density to integrate up to one are slightly different from those used in MGCI. In 
particular $c_{i}=k_{i}-1$-see Eqs. (32) and (38).The bivariate p.d.f. of the MGCII in terms of the variables $x_{t}$ and $y_{t}$ is:

$F_{I I}\left(x_{t}, y_{t}\right)=\frac{1}{3} G\left(x_{t}, y_{t}\right)+\frac{1}{3} g\left(x_{t}\right) g\left(y_{t}\right)\left[\frac{1}{c_{x}}\left[\sum_{s=1}^{q} d_{s x} H_{s}\left(x_{t}\right)\right]^{2}+\frac{1}{c_{y}}\left[\sum_{s=1}^{q} d_{s y} H_{s}\left(y_{t}\right)\right]^{2}\right]$

where

$$
\begin{gathered}
c_{x}=\int g\left(x_{t}\right)\left[\sum_{s=1}^{q} d_{s i} H_{s}\left(x_{t}\right)\right]^{2} d x_{t}=\sum_{s=1}^{q} d_{s x}^{2} s ! \text { and } \\
c_{y}=\int g\left(y_{t}\right)\left[\sum_{s=1}^{q} d_{s i} H_{s}\left(y_{t}\right)\right]^{2} d y_{t}=\sum_{s=1}^{q} d_{s y}^{2} s !
\end{gathered}
$$

For this density it is also easy to check that it integrates up to one and that the marginal density of $x_{t}$, for example, is distributed according to (42) (see Proof 3 in the Appendix).

$$
f_{I I}\left(x_{t}\right)=\frac{2}{3} g\left(x_{t}\right)+\frac{1}{3 c_{x}} g\left(x_{t}\right)\left[\sum_{s=1}^{q} d_{s x} H_{s}\left(x_{t}\right)\right]^{2}
$$

All these densities may be considered as "standard" in the sense that their corresponding moments depend only on the whole sequence of parameters $\left\{d_{s i}\right\}$. Nevertheless, depending on the characteristics of the underlying variables, alternative specifications can be achieved by either constraining 
the density to fixed values (for example symmetry may often be achieved by restricting the odd parameters to zero) or doing transformations of the original variables (in order to obtain different means or variances). In particular, the bivariate Edgeworth-Sargan density - a transformed version of (30) - with variances $\sigma_{x}^{2}\left(1+2 d_{2 x}\right)$ and $\sigma_{y}^{2}\left(1+2 d_{2 y}\right)$ and correlation coefficient $\rho_{x y}$ can be written as

$F\left(x_{t}, y_{t}\right)=G^{*}\left(x_{t}, y_{t}\right)+\frac{1}{\sigma_{x} \sigma_{y}} g\left(\frac{x_{t}}{\sigma_{x}}\right) g\left(\frac{y_{t}}{\sigma_{y}}\right)\left[\sum_{s=1}^{q} d_{s x} H_{s}\left(\frac{x_{t}}{\sigma_{x}}\right)+\sum_{s=1}^{q} d_{s y} H_{s}\left(\frac{y_{t}}{\sigma_{y}}\right)\right]$

where

$G^{*}\left(x_{t}, y_{t}\right)=\frac{1}{2 \pi \sigma_{x} \sigma_{y} \sqrt{1-\rho_{x y}^{2}}} \exp \left\{\frac{1}{2\left(1-\rho_{x y}^{2}\right)}\left[\left(\frac{x_{t}}{\sigma_{x}}\right)^{2}+\left(\frac{y_{t}}{\sigma_{y}}\right)^{2}-2 \rho_{x y} \frac{x_{y} y_{t}}{\sigma_{x} \sigma_{y}}\right]\right\}$

Alternatively, a positive version of the bivariate distribution shown in (33) with variances proportional to $\sigma_{x}^{2}$ and $\sigma_{y}^{2}$ and correlation coefficient $\rho_{x y}$ can be expressed as

$$
\begin{aligned}
F_{I}\left(x_{t}, y_{t}\right)= & \frac{1}{3} G^{*}\left(x_{t}, y_{t}\right)+\frac{1}{3 \sigma_{x} \sigma_{y}} g\left(\frac{x_{t}}{\sigma_{x}}\right) g\left(\frac{y_{t}}{\sigma_{y}}\right) \frac{1}{k_{x}}\left[1+\sum_{s=1}^{q} d_{s x} H_{s}\left(\frac{x_{t}}{\sigma_{x}}\right)\right]^{2} \\
& +\frac{1}{3 \sigma_{x} \sigma_{y}} g\left(\frac{x_{t}}{\sigma_{x}}\right) g\left(\frac{y_{t}}{\sigma_{y}}\right) \frac{1}{k_{y}}\left[1+\sum_{s=1}^{q} d_{s y} H_{s}\left(\frac{y_{t}}{\sigma_{y}}\right)\right]^{2}
\end{aligned}
$$

where $G^{*}\left(x_{t}, y_{t}\right)$ is the multivariate normal - see Eq. (44), and $k_{x}$ and $k_{y}$ are the constants displayed in Eqs. (34) and (35). Moreover, conditional 
variance and kurtosis - see Eqs. (21) and (22) - can be also introduced into multivariate Gram-Charlier models as shown in Eqs. (46) to (51), which provide the bivariate generalisation of the univariate density displayed in Eq. (23).

$$
\begin{aligned}
F\left(x_{t}, y_{t}\right)= & G^{+}\left(x_{t}, y_{t}\right)+\frac{1}{\sigma_{x t} \sigma_{y t}} g\left(\frac{x_{t}}{\sigma_{x t}}\right) g\left(\frac{y_{t}}{\sigma_{y t}}\right) \frac{k_{x t}^{4}-3}{24}\left[\left(\frac{x_{t}}{\sigma_{x t}}\right)^{4}+6\left(\frac{x_{t}}{\sigma_{x t}}\right)^{2}+3\right] \\
& +\frac{1}{\sigma_{x t} \sigma_{y t}} g\left(\frac{x_{t}}{\sigma_{x t}}\right) g\left(\frac{y_{t}}{\sigma_{y t}}\right) \frac{k_{y t}^{4}-3}{24}\left[\left(\frac{y_{t}}{\sigma_{y t}}\right)^{4}+6\left(\frac{y_{t}}{\sigma_{y t}}\right)^{2}+3\right] \\
G^{+}\left(x_{t}, y_{t}\right)= & \frac{1}{2 \pi \sigma_{x t} \sigma_{y t} \sqrt{1-\rho_{x y}^{2}}} \exp \left\{\frac{1}{2\left(1-\rho_{x y}^{2}\right)}\left[\left(\frac{x_{t}}{\sigma_{x t}}\right)^{2}+\left(\frac{y_{t}}{\sigma_{y t}}\right)^{2}-2 \rho_{x y} \frac{x_{y} y_{t}}{\sigma_{x t} \sigma_{y t}}\right]\right\}
\end{aligned}
$$

$$
\sigma_{x t}^{2}=\alpha_{0 x}+\alpha_{1 x} x_{t-1}^{2}+\alpha_{2 x} \sigma_{x, t-1}^{2}
$$

$$
\sigma_{y t}^{2}=\alpha_{0 y}+\alpha_{1 y} y_{t-1}^{2}+\alpha_{2 y} \sigma_{y, t-1}^{2}
$$

$$
\begin{aligned}
& k_{x t}^{4}=\beta_{0 x}+\beta_{1 x}\left(\frac{x_{t-1}}{\sigma_{x, t-1}}\right)^{4}+\beta_{2} k_{x, t-1}^{4} \\
& k_{y t}^{4}=\beta_{0 y}+\beta_{1 y}\left(\frac{y_{t-1}}{\sigma_{y, t-1}}\right)^{4}+\beta_{2} k_{y, t-1}^{4}
\end{aligned}
$$




\section{EMPIRICAL EXAMPLES}

In this section we estimate by maximum likelihood the parameters of some of the densities described in previous sections. The data used for the density estimation correspond to the residuals of the previous estimation of a first order autoregressive process for the continuously compounded returns of the corresponding stock index (measured as the difference of logarithms). Therefore, we consider that the conditional mean of each variable is generated by an $\mathrm{AR}(1)$ process and the conditional variance is supposed to be either constant or $\operatorname{GARCH}(1,1)$ - note that estimating the density for the residuals of the $\mathrm{AR}(1)$ involves a loss in efficiency (not in consistency) but also simplifies the convergence of the optimisation algorithms. Table 1 shows the estimates for some univariate Gram-Charlier densities including two specifications - these of (5) and (8) - with either constant or conditional GARCH(1,1) variances. Table 2 displays the bivariate densities corresponding to the univariate distributions shown in Table 1, i.e. the MES density -see Eq. (43) - and some of its transformations defined in this paper to avoid negative values - a scaled version of the MGCI described in Eq. (33), whose marginal density of $x$ is that of equation (36). All these densities were truncated on the eighth Hermite polynomial and all the odd parameters were dropped after the corresponding constraints had been tested. The densities were estimated using daily data from the Dow Jones and the FTSE indices spanning from January 1970 to May 1996. The columns of the tables are numbered according to the different density specification and data used (see the details below). 
I: Edgeworth-Sargan density - type (5) - with constant variance for the Dow Jones index.

II: Positive univariate density - type (8) - with constant variance for the Dow Jones index.

III: Positive univariate density - type (8) - with $\operatorname{GARCH}(1,1)$ variance for the Dow Jones index.

IV: Edgeworth-Sargan density - type (5) - with constant variance for the FTSE index.

V: Positive univariate density - type (8) - with constant variance for the FTSE index.

VI: Positive univariate density - type (8) - with $\operatorname{GARCH}(1,1)$ variance for the FTSE index.

VII: Bivariate Edgeworth-Sargan density - type (43) - with constant variances for the Dow Jones $(x)$ and the FTSE $(y)$ indices.

VIII: Bivariate MGCI density - type (33) - with constant variances for the Dow Jones $(x)$ and the FTSE $(y)$ indices.

IX: Bivariate Edgeworth-Sargan density - type (43) - with $\operatorname{GARCH}(1,1)$ variances for the Dow Jones $(x)$ and the FTSE $(y)$ indices.

X: Bivariate MGCI density - type (33) - with $\mathrm{GARCH}(1,1)$ variances for the Dow Jones $(x)$ and the FTSE $(y)$ indices.

\section{Insert Tables 1 and 2}

Both tables also display the corresponding t-ratio statistics for each parameter (in parentheses), the log-likelihood value $(\ln L)$ and the Schwarz 
Bayesian Information Criterion (B.I.C.), defined as B.I.C. $=-\ln L+$ $p \ln (T) / 2$ where $p$ stands for the number of the parameters of the model. It must be noted that most of the parameters of the models considered are significant at 5 per cent confidence level, thus, the models seem to be accurate measures of the density underlying the Dow Jones and the FTSE indices. However, the values of the parameters are quite different since the Edgeworth-Sargan densities need all the parameters to be positive (except $d_{2}$ ) whilst the other specifications, such as the MGCI, are always positive whichever the sign of the parameters. Moreover, both the $\ln L$ and the B.I.C. criteria throw evidence in favour of the Edgeworth-Sargan representations as compared to other Gram-Charlier densities. This evidence highlights the fact that "positive Gram-Charlier expansions" can be adequate representations for both univariate and multivariate densities but at the cost of a loss of accuracy when compared to other simpler Edgeworth-Sargan representations. Nevertheless, the use of Edgeworth-Sargan densities requires a more careful selection of initial values (or even implementing some constrains to the parameters) to avoid the problems caused by possible negative values.

Moreover, the consideration of $\operatorname{GARCH}(1,1)$ processes improves the estimates for the conditional densities, which enforces the idea of considering other conditional moments that can be incorporated to this kind of densities. Despite this idea sounds promising, it also provokes several shortcomings from both a theoretical and an empirical point of view (some examples were commented in Section 3 raising the fact that the succesful introduction of conditional moments is sensible to the density specification). Even more, the interpretation of the parameters is also sensible to the density specification 
and thus the estimates of the parameters must be analysed accordingly. For example, the naïve interpretation of $\rho_{x y}$ as the correlation coefficient among both indices (note that $\rho_{x y}$ stands for the correlation coefficient among both variables only for the MES density and provided that $d_{2 x}=d_{2 y}=0$ ) is clearly misleading, as revealed by the the sharp increase of this parameter when using the MGCI instead of the MES, which seems to be rather puzzling. The consideration of all these issues play an important role when the estimated densities are used to achieve more accurate measures of risk.

\section{CONCLUDING REMARKS}

This paper presents different specifications for densities based on GramCharlier and Edgeworth series in both a univariate and a multivariate framework. The focus of the paper is done on guaranteeing positive definiteness by using the orthogonality properties of the Hermite polynomials and extending some of the proposals given in the literature to a multivariate context. These densities are shown to adequately fit most high frequency data but the gains in terms of accuracy are not clear compared to the Edgeworth-Sargan and its multivariate version defined in Perote (2004), which is not strictly a density from a theoretical point of view due to potential negative values. Therefore in contexts where strong presence of outliers is perceived or when the recursive algorithms to obtain systematically estimates are needed (as in forecasting exercises) the use of these positive densities are strongly recommended. The paper also emphasises the fact that there exist many different positive specifications of the densities based on Hermite 
polynomials, and that the selection among all of them should be based on the econometric assumptions used to model the data. For example, if the model involves the study of other conditional moments than the conditional variance, it is not always possible to guarantee positive definiteness of the conditional density. In this sense, the interpretation of the density parameters in terms of the density moments is the key to define densities based on Gram-Charlier and Edgeworth series capable of improving data fits and thus representing more accurate measures of risk.

Acknowledgement 1 This paper was written whilst both authors were visiting the Department of Economics of the London School of Economics and the Judge Institute of Management of the University of Cambridge, respectively. We would like to thank Javier Hidalgo for his support and Tim Bollerslev for his helpful comments. Responsibility for the contents remains solely with the authors. 


\section{APPENDIX: PROOFS}

\section{Proof 1:}

$$
\begin{aligned}
k & =\int\left[1+\sum_{s=1}^{q} d_{s} H_{s}(x)\right]^{2} g(x) d x \\
& =\int g(x) d x+\int\left[\sum_{s=1}^{q} d_{s} H_{s}(x)\right]^{2} g(x) d x+2 \sum_{s=1}^{q} d_{s} \int H_{s}(x) g(x) d x \\
& =\int g(x) d x+\int\left[\sum_{s=1}^{q} d_{s} H_{s}(x)\right]^{2} g(x) d x=1+\sum_{s=1}^{q} \sum_{j=1}^{q} d_{s} d_{j} \int H_{s}(x) H_{j}(x) g(x) d x \\
& =1+\sum_{s=1}^{q} d_{s}^{2} \int H_{s}(x)^{2} g(x) d x=\int\left[1+\sum_{s=1}^{q} d_{s}^{2} H_{s}(x)^{2}\right] g(x) d x=1+\sum_{s=1}^{q} d_{s}^{2} s !
\end{aligned}
$$

\section{Proof 2:}

$$
\begin{aligned}
\mathbf{E}_{t-1}\left[\varepsilon_{t}^{2}\right] & =\int \varepsilon_{t}^{2} f_{1}\left(\varepsilon_{t}\right) d \varepsilon_{t}=\int \varepsilon_{t}^{2} g\left(\varepsilon_{t}\right)\left[1+d_{4 t}\left(\varepsilon_{t}^{4}-6 \varepsilon_{t}^{2}+3\right)\right] d \varepsilon_{t} \\
& =\int \varepsilon_{t}^{2} g\left(\varepsilon_{t}\right) d \varepsilon_{t}+d_{4 t} \int\left(\varepsilon_{t}^{6}-6 \varepsilon_{t}^{4}+3 \varepsilon_{t}^{2}\right) g\left(\varepsilon_{t}\right) d \varepsilon_{t}=1 \\
\mathbf{E}_{t-1}\left[\varepsilon_{t}^{4}\right] & =\int \varepsilon_{t}^{4} f_{1}(x) d \varepsilon_{t}=\int \varepsilon_{t}^{4} g\left(\varepsilon_{t}\right)\left[1+d_{4 t}\left(\varepsilon_{t}^{4}-6 \varepsilon_{t}^{2}+3\right)\right] d \varepsilon_{t} \\
& =\int \varepsilon_{t}^{4} g\left(\varepsilon_{t}\right) d \varepsilon_{t}+d_{4 t} \int\left(\varepsilon_{t}^{8}-6 \varepsilon_{t}^{6}+3 \varepsilon_{t}^{4}\right) g\left(\varepsilon_{t}\right) d \varepsilon_{t}=3+24 d_{4 t}
\end{aligned}
$$




$$
\begin{aligned}
& E_{t-1}\left[\varepsilon_{t}^{2}\right]=\int \varepsilon_{t}^{2} f_{3}\left(\varepsilon_{t}\right) d \varepsilon_{t}=\int \varepsilon_{t}^{2} \frac{1}{1+24 d_{4 t}^{2}} g\left(\varepsilon_{t}\right)\left[1+d_{4 t}\left(\varepsilon_{t}^{4}-6 \varepsilon_{t}^{2}+3\right)\right]^{2} d \varepsilon_{t} \\
& =\frac{1}{1+24 d_{4 t}^{2}}\left[\int \varepsilon_{t}^{2} g\left(\varepsilon_{t}\right)\left[1+d_{4 t}^{2}\left(\varepsilon_{t}^{4}-6 \varepsilon_{t}^{2}+3\right)^{2}+2 d_{4 t}\left(\varepsilon_{t}^{4}-6 \varepsilon_{t}^{2}+3\right)\right] d \varepsilon_{t}\right. \\
& =\frac{1}{1+24 d_{4 t}^{2}}\left[\int \varepsilon_{t}^{2} g\left(\varepsilon_{t}\right) d \varepsilon_{t}+d_{4 t}^{2} \int \varepsilon_{t}^{2}\left(\varepsilon_{t}^{8}-12 \varepsilon_{t}^{6}+42 \varepsilon_{t}^{4}-36 \varepsilon_{t}^{2}+9\right) g\left(\varepsilon_{t}\right) d \varepsilon_{t}\right. \\
& \left.+2 d_{4 t} \int \varepsilon_{t}^{2}\left(\varepsilon_{t}^{4}-6 \varepsilon_{t}^{2}+3\right) g\left(\varepsilon_{t}\right) d \varepsilon_{t}\right] \\
& =\frac{1}{1+24 d_{4 t}^{2}}\left[\int \varepsilon_{t}^{2} g\left(\varepsilon_{t}\right) d \varepsilon_{t}+d_{4 t}^{2} \int\left(\varepsilon_{t}^{10}-12 \varepsilon_{t}^{8}+42 \varepsilon_{t}^{6}-36 \varepsilon_{t}^{4}+9 \varepsilon_{t}^{2}\right) g\left(\varepsilon_{t}\right) d \varepsilon_{t}\right. \\
& \left.+2 d_{4 t} \int\left(\varepsilon_{t}^{6}-6 \varepsilon_{t}^{4}+3 \varepsilon_{t}^{2}\right) g\left(\varepsilon_{t}\right) d \varepsilon_{t}\right]=\frac{1+216 d_{4 t}^{2}}{1+24 d_{4 t}^{2}}
\end{aligned}
$$

$$
\begin{aligned}
& E_{t-1}\left[\varepsilon_{t}^{4}\right]=\int \varepsilon_{t}^{4} f_{3}\left(\varepsilon_{t}\right) d \varepsilon_{t}=\int \varepsilon_{t}^{4} \frac{1}{1+24 d_{4 t}^{2}} g\left(\varepsilon_{t}\right)\left[1+d_{4 t}\left(\varepsilon_{t}^{4}-6 \varepsilon_{t}^{2}+3\right)\right]^{2} d \varepsilon_{t} \\
& =\frac{1}{1+24 d_{4 t}^{2}}\left[\int \varepsilon_{t}^{4} g\left(\varepsilon_{t}\right)\left[1+d_{4 t}^{2}\left(\varepsilon_{t}^{4}-6 \varepsilon_{t}^{2}+3\right)^{2}+2 d_{4 t}\left(\varepsilon_{t}^{4}-6 \varepsilon_{t}^{2}+3\right)\right] d \varepsilon_{t}\right. \\
& =\frac{1}{1+24 d_{4 t}^{2}}\left[\int \varepsilon_{t}^{4} g\left(\varepsilon_{t}\right) d \varepsilon_{t}+d_{4 t}^{2} \int \varepsilon_{t}^{4}\left(\varepsilon_{t}^{8}-12 \varepsilon_{t}^{6}+42 \varepsilon_{t}^{4}-36 \varepsilon_{t}^{2}+9\right) g\left(\varepsilon_{t}\right) d \varepsilon_{t}\right. \\
& \left.+2 d_{4 t} \int \varepsilon_{t}^{4}\left(\varepsilon_{t}^{4}-6 \varepsilon_{t}^{2}+3\right) g\left(\varepsilon_{t}\right) d \varepsilon_{t}\right] \\
& =\frac{1}{1+24 d_{4 t}^{2}}\left[\int \varepsilon_{t}^{4} g\left(\varepsilon_{t}\right) d \varepsilon_{t}+d_{4 t}^{2} \int\left(\varepsilon_{t}^{12}-12 \varepsilon_{t}^{10}+42 \varepsilon_{t}^{8}-36 \varepsilon_{t}^{6}+9 \varepsilon_{t}^{4}\right) g\left(\varepsilon_{t}\right) d \varepsilon_{t}\right. \\
& \left.+2 d_{4 t} \int\left(\varepsilon_{t}^{8}-6 \varepsilon_{t}^{6}+3 \varepsilon_{t}^{4}\right) g\left(\varepsilon_{t}\right) d \varepsilon_{t}\right]=\frac{3+2952 d_{4 t}^{2}+6 d_{4 t}}{1+24 d_{4 t}^{2}}
\end{aligned}
$$

$$
\begin{aligned}
& E_{t-1}\left[\varepsilon_{t}^{2}\right]=\int \varepsilon_{t}^{2} f_{4}\left(\varepsilon_{t}\right) d \varepsilon_{t}=\int \varepsilon_{t}^{2} \frac{1}{1+24 d_{4}^{2}} g\left(\varepsilon_{t}\right)\left[1+d_{4 t}^{2}\left(\varepsilon_{t}^{4}-6 \varepsilon_{t}^{2}+3\right)\right]^{2} d \varepsilon_{t} \\
& =\frac{1}{1+24 d_{4 t}^{2}}\left[\int \varepsilon_{t}^{2} g\left(\varepsilon_{t}\right) d \varepsilon_{t}+d_{4 t}^{2} \int \varepsilon_{t}^{2}\left(\varepsilon_{t}^{8}-12 \varepsilon_{t}^{6}+42 \varepsilon_{t}^{4}-36 \varepsilon_{t}^{2}+9\right) g\left(\varepsilon_{t}\right) d \varepsilon_{t}\right] \\
& =\frac{1}{1+24 d_{4 t}^{2}}\left[\int \varepsilon_{t}^{2} g\left(\varepsilon_{t}\right) d \varepsilon_{t}+d_{4 t}^{2} \int\left(\varepsilon_{t}^{10}-12 \varepsilon_{t}^{8}+42 \varepsilon_{t}^{6}-36 \varepsilon_{t}^{4}+9 \varepsilon_{t}^{2}\right) g\left(\varepsilon_{t}\right) d \varepsilon_{t}\right]=\frac{1+216 d_{4 t}^{2}}{1+24 d_{4 t}^{2}}
\end{aligned}
$$




$$
\begin{aligned}
& E_{t-1}\left[\varepsilon_{t}^{4}\right]=\int \varepsilon_{t}^{4} f_{4}\left(\varepsilon_{t}\right) d \varepsilon_{t}=\int \varepsilon_{t}^{4} \frac{1}{1+24 d_{4}^{2}} g\left(\varepsilon_{t}\right)\left[1+d_{4 t}^{2}\left(\varepsilon_{t}^{4}-6 \varepsilon_{t}^{2}+3\right)\right]^{2} d \varepsilon_{t} \\
& =\frac{1}{1+24 d_{4 t}^{2}}\left[\int \varepsilon_{t}^{4} g\left(\varepsilon_{t}\right) d \varepsilon_{t}+d_{4 t}^{2} \int \varepsilon_{t}^{4}\left(\varepsilon_{t}^{8}-12 \varepsilon_{t}^{6}+42 \varepsilon_{t}^{4}-36 \varepsilon_{t}^{2}+9\right) g\left(\varepsilon_{t}\right) d \varepsilon_{t}\right] \\
& =\frac{1}{1+24 d_{4 t}^{2}}\left[\int \varepsilon_{t}^{4} g\left(\varepsilon_{t}\right) d \varepsilon_{t}+d_{4 t}^{2} \int\left(\varepsilon_{t}^{12}-12 \varepsilon_{t}^{10}+42 \varepsilon_{t}^{8}-36 \varepsilon_{t}^{6}+9 \varepsilon_{t}^{4}\right) g\left(\varepsilon_{t}\right) d \varepsilon_{t}\right]=\frac{3+2952 d_{4 t}^{2}}{1+24 d_{4 t}^{2}}
\end{aligned}
$$

\section{Proof 3:}

$$
\begin{aligned}
& \int F\left(x_{t}, y_{t}\right) d y_{t}=\int G\left(x_{t}, y_{t}\right) d y+g\left(x_{t}\right) \sum_{s=1}^{q} d_{s x} H_{s}\left(x_{t}\right) \int g\left(y_{t}\right) d y_{t} \\
& +g\left(x_{t}\right) \sum_{s=1}^{q} d_{s y} \int g\left(y_{t}\right) H_{s}\left(y_{t}\right) d y_{t} \\
& =g\left(x_{t}\right)\left[1+\sum_{s=1}^{q} d_{s x} H_{s}\left(x_{t}\right)\right]=f\left(x_{t}\right) \\
& \iint F\left(x_{t}, y_{t}\right) d y_{t} d x_{t}=\int\left[1+\sum_{s=1}^{q} d_{s x} H_{s}\left(x_{t}\right)\right] g\left(x_{t}\right) d x_{t}=1 \\
& \int F_{I}\left(x_{t}, y_{t}\right) d y_{t}=\frac{1}{3} \int G\left(x_{t}, y_{t}\right) d y_{t}+\frac{1}{3 k_{x}} g\left(x_{t}\right)\left[1+\sum_{s=1}^{q} d_{s x} H_{s}\left(x_{t}\right)\right]^{2} \int g\left(y_{t}\right) d y_{t} \\
& +\frac{1}{3 k_{y}} g\left(x_{t}\right) \int\left[1+\sum_{s=1}^{q} d_{s y} H_{s}\left(y_{t}\right)\right]^{2} g\left(y_{t}\right) d y_{t} \\
& =\frac{2}{3} g\left(x_{t}\right)+\frac{1}{3 k_{x}} g\left(x_{t}\right)\left[1+\sum_{s=1}^{q} d_{s x} H_{s}\left(x_{t}\right)\right]^{2}=f_{I}\left(x_{t}\right) \\
& \iint F_{I}\left(x_{t}, y_{t}\right) d y_{t} d x_{t}=\frac{2}{3} \int g\left(x_{t}\right) d x_{t}+\frac{1}{3 k_{x}} \int g\left(x_{t}\right)\left[1+\sum_{s=1}^{q} d_{s x} H_{s}\left(x_{t}\right)\right]^{2} d x_{t}=\frac{2}{3}+\frac{1}{3}=1
\end{aligned}
$$




$$
\begin{aligned}
\int F_{I I}\left(x_{t}, y_{t}\right) d y_{t} & =\frac{1}{3} \int G\left(x_{t}, y_{t}\right) d y_{t}+\frac{1}{3 c_{x}} g\left(x_{t}\right)\left[\sum_{s=1}^{q} d_{s x} H_{s}\left(x_{t}\right)\right]^{2} \int g\left(y_{t}\right) d y_{t} \\
& +\frac{1}{3 c_{y}} g\left(x_{t}\right) \int\left[\sum_{s=1}^{q} d_{s y} H_{s}\left(y_{t}\right)\right]^{2} g\left(y_{t}\right) d y_{t} \\
& =\frac{2}{3} g\left(x_{t}\right)+\frac{1}{3 c_{x}} g\left(x_{t}\right)\left[\sum_{s=1}^{q} d_{s x} H_{s}\left(x_{t}\right)\right]^{2}=f_{I I}\left(x_{t}\right) \\
\iint F_{I I}\left(x_{t}, y_{t}\right) d y_{t} d x_{t} & =\frac{2}{3} \int g\left(x_{t}\right) d x_{t}+\frac{1}{3 c_{x}} \int g\left(x_{t}\right)\left[\sum_{s=1}^{q} d_{s x} H_{s}\left(x_{t}\right)\right]^{2} d x_{t}=\frac{2}{3}+\frac{1}{3}=1
\end{aligned}
$$


TABLE 1: Univariate Edgeworth and Gram-Charlier densities.

\begin{tabular}{|c|c|c|c|c|c|c|}
\hline & I & II & III & IV & $\mathbf{V}$ & $\overline{\text { VI }}$ \\
\hline$\widehat{\sigma}_{x}$ & $\begin{array}{c}.96 \times 10^{-2} \\
(40.62)\end{array}$ & $\begin{array}{c}.68 \times 10^{-2} \\
(76.48)\end{array}$ & & & & \\
\hline$\widehat{d}_{2 x}$ & $\begin{array}{c}-.69 \times 10^{-1} \\
(-3.31)\end{array}$ & $\begin{array}{c}.61 \times 10^{-1} \\
(7.95)\end{array}$ & $\begin{array}{l}.27 \\
(53.38)\end{array}$ & & & \\
\hline$\widehat{d}_{4 x}$ & $\begin{array}{c}.72 \times 10^{-1} \\
(10.71)\end{array}$ & $\begin{array}{c}.71 \times 10^{-2} \\
(3.66)\end{array}$ & $\begin{array}{c}.32 \times 10^{-1} \\
(23.44)\end{array}$ & & & \\
\hline$\widehat{d}_{6 x}$ & $\begin{array}{c}.75 \times 10^{-2} \\
(4.11)\end{array}$ & $\begin{array}{c}-.51 \times 10^{-2} \\
(-16.06)\end{array}$ & $\begin{array}{c}-.65 \times 10^{-2} \\
(-28.55) \\
\end{array}$ & & & \\
\hline$\widehat{d}_{8 x}$ & $\begin{array}{c}.14 \times 10^{-2} \\
(7.44)\end{array}$ & $\begin{array}{c}-.42 \times 10^{-3} \\
(-8.78)\end{array}$ & $\begin{array}{c}-.15 \times 10^{-2} \\
(-45.61)\end{array}$ & & & \\
\hline$\widehat{\sigma}_{y}$ & & & & $\begin{array}{c}.11 \times 10^{-1} \\
(40.13)\end{array}$ & $\begin{array}{c}.91 \times 10^{-2} \\
(56.64)\end{array}$ & \\
\hline$\widehat{d}_{2 y}$ & & & & $\begin{array}{l}-.15 \\
(-8.68)\end{array}$ & $\begin{array}{c}.24 \times 10^{-1} \\
(2.66)\end{array}$ & $\begin{array}{l}.31 \\
(56.71)\end{array}$ \\
\hline$\widehat{d}_{4 y}$ & & & & $\begin{array}{c}.75 \times 10^{-1} \\
(11.99)\end{array}$ & $\begin{array}{l}.12 \\
(8.34)\end{array}$ & $\begin{array}{l}.48 \times 10^{-1} \\
(29.10)\end{array}$ \\
\hline$\widehat{d}_{6 y}$ & & & & $\begin{array}{c}.56 \times 10^{-2} \\
(3.24)\end{array}$ & $\begin{array}{c}-.35 \times 10^{-2} \\
(-9.78)\end{array}$ & $\begin{array}{c}.72 \times 10^{-3} \\
(2.34)\end{array}$ \\
\hline$\widehat{d}_{8 y}$ & & & & $\begin{array}{c}.13 \times 10^{-2} \\
(7.17)\end{array}$ & $\begin{array}{c}-.29 \times 10^{-3} \\
(-6.74)\end{array}$ & $\begin{array}{c}.11 \times 10^{-2} \\
(-37.78)\end{array}$ \\
\hline$\widehat{\alpha}_{0 x}$ & & & $\begin{array}{c}.15 \times 10^{-6} \\
(64.44)\end{array}$ & & & \\
\hline$\widehat{\alpha}_{1 x}$ & & & $\begin{array}{l}.97 \\
(2084.42)\end{array}$ & & & \\
\hline$\widehat{\alpha}_{2 x}$ & & & $\begin{array}{l}.53 \times 10^{-2} \\
(138.16)\end{array}$ & & & \\
\hline$\widehat{\alpha}_{0 y}$ & & & & & & $\begin{array}{c}56 \times 10^{-6} \\
(79.94)\end{array}$ \\
\hline$\widehat{\alpha}_{1 y}$ & & & & & & $\begin{array}{l}.89 \\
(2297.25)\end{array}$ \\
\hline$\widehat{\alpha}_{2 y}$ & & & & & & $\begin{array}{c}.14 \times 10^{-1} \\
(93.89)\end{array}$ \\
\hline $\ln T$ & 24800.9 & 24628.4 & 62351.7 & 24351.8 & 24312.6 & 61818.5 \\
\hline B.I.C. & -24778.8 & -24606.3 & -62320.8 & -24329.8 & -24290.5 & -61787.6 \\
\hline
\end{tabular}

t-ratios in parentheses. ${ }^{*}$ Non significant at $5 \%$ confidence level. 
TABLE 2: Multivariate Edgeworth and Gram-Charlier densities.

\begin{tabular}{|c|c|c|c|c|}
\hline & $\overline{\text { VII }}$ & VIII & IX & $\mathbf{X}$ \\
\hline$\widehat{\sigma}_{x}$ & $\begin{array}{c}.78 \times 10^{-2} \\
(51.85)\end{array}$ & $\begin{array}{c}.93 \times 10^{-2} \\
(64.19)\end{array}$ & & \\
\hline$\widehat{d}_{2 x}$ & $\begin{array}{l}.27 \\
(6.02)\end{array}$ & $\begin{array}{l}-.25 \\
(-5.42)\end{array}$ & $\begin{array}{c}-.44 \times 10^{-2} \\
(.06)^{*}\end{array}$ & $\begin{array}{l}-.32 \\
(-10.21)\end{array}$ \\
\hline$\widehat{d}_{4 x}$ & $\begin{array}{l}.19 \\
(8.61)\end{array}$ & $\begin{array}{c}.51 \times 10^{-1} \\
(5.36)\end{array}$ & $\begin{array}{c}.83 \times 10^{-1} \\
(4.99)\end{array}$ & $\begin{array}{c}.25 \times 10^{-1} \\
(3.49)\end{array}$ \\
\hline$\widehat{d}_{6 x}$ & $\begin{array}{c}.34 \times 10^{-1} \\
(6.28)\end{array}$ & $\begin{array}{c}-.14 \times 10^{-1} \\
(-10.00)\end{array}$ & $\begin{array}{c}.11 \times 10^{-1} \\
(2.10)\end{array}$ & $\begin{array}{c}-.22 \times 10^{-2} \\
(-2.96)\end{array}$ \\
\hline$\widehat{d}_{8 x}$ & $\begin{array}{c}.28 \times 10^{-2} \\
(6.82)\end{array}$ & $\begin{array}{c}.63 \times 10^{-3} \\
(4.82)\end{array}$ & $\begin{array}{c}.16 \times 10^{-2} \\
(3.45)\end{array}$ & $\begin{array}{c}.44 \times 10^{-3} \\
(5.41)\end{array}$ \\
\hline$\widehat{\sigma}_{y}$ & $\begin{array}{c}.11 \times 10^{-1} \\
(39.89)\end{array}$ & $\begin{array}{c}.90 \times 10^{-2} \\
(68.23)\end{array}$ & & \\
\hline$\widehat{d}_{2 y}$ & $\begin{array}{l}-.26 \\
(-8.37)\end{array}$ & $\begin{array}{c}-.35 \times 10^{-1} \\
(-1.36)^{*}\end{array}$ & $\begin{array}{c}.42 \times 10^{-1} \\
(.55)^{*}\end{array}$ & $\begin{array}{c}-.33 \times 10^{-1} \\
(-1.25)^{*}\end{array}$ \\
\hline$\widehat{d}_{4 y}$ & $\begin{array}{l}.12 \\
(11.15)\end{array}$ & $\begin{array}{c}.43 \times 10^{-1} \\
(9.51)\end{array}$ & $\begin{array}{c}.44 \times 10^{-1} \\
(3.37)\end{array}$ & $\begin{array}{c}.18 \times 10^{-1} \\
(6.03)\end{array}$ \\
\hline$\widehat{d}_{6 y}$ & $\begin{array}{c}.84 \times 10^{-2} \\
(2.92)\end{array}$ & $\begin{array}{c}-.54 \times 10^{-2} \\
(-5.13)\end{array}$ & $\begin{array}{c}.77 \times 10^{-2} \\
(1.72)^{*}\end{array}$ & $\begin{array}{c}.05 \times 10^{-2} \\
(0.93)^{*}\end{array}$ \\
\hline$\widehat{d}_{8 y}$ & $\begin{array}{c}.23 \times 10^{-2} \\
(7.96)\end{array}$ & $\begin{array}{c}-.62 \times 10^{-3} \\
(-7.13)\end{array}$ & $\begin{array}{c}.53 \times 10^{-3} \\
(1.49)^{*}\end{array}$ & $\begin{array}{c}.67 \times 10^{-4} \\
(1.41)^{*}\end{array}$ \\
\hline$\widehat{\alpha}_{0 x}$ & & & $\begin{array}{c}.64 \times 10^{-6} \\
(3.14)\end{array}$ & $\begin{array}{c}.71 \times 10^{-6} \\
(3.31)\end{array}$ \\
\hline$\widehat{\alpha}_{1 x}$ & & & $\begin{array}{l}.96 \\
(114.48)\end{array}$ & $\begin{array}{l}.96 \\
(118.23)\end{array}$ \\
\hline$\widehat{\alpha}_{2 x}$ & & & $\begin{array}{c}.41 \times 10^{-1} \\
(6.68)\end{array}$ & $\begin{array}{l}.0 .5 \\
(8.24)\end{array}$ \\
\hline$\widehat{\alpha}_{0 y}$ & & & $\begin{array}{c}.13 \times 10^{-5} \\
(4.97)\end{array}$ & $\begin{array}{c}.14 \times 10^{-5} \\
(5.08)\end{array}$ \\
\hline$\widehat{\alpha}_{1 y}$ & & & $\begin{array}{l}.91 \\
(98.54)\end{array}$ & $\begin{array}{l}.91 \\
(97.12)\end{array}$ \\
\hline$\widehat{\alpha}_{2 y}$ & & & $\begin{array}{c}.82 \times 10^{-1} \\
(7.92)\end{array}$ & $\begin{array}{c}.87 \times 10^{-1} \\
(9.98)\end{array}$ \\
\hline$\widehat{\rho}_{x y}$ & $\begin{array}{l}.09 \\
(8.71)\end{array}$ & $\begin{array}{l}.43 \\
(15.31)\end{array}$ & $\begin{array}{l}.18 \\
(14.05)\end{array}$ & $\begin{array}{l}.65 \\
(23.43)\end{array}$ \\
\hline $\ln L$ & 49178.8 & 44885.0 & 50088.7 & 45200.5 \\
\hline B.I.C. & -49130.2 & -44836.5 & -50022.4 & -45134.3 \\
\hline
\end{tabular}

t-ratios in parentheses. ${ }^{*}$ Non significant at $5 \%$ confidence level. 


\section{References}

[1] Barton D.E. and Dennis K.E.R. (1952), "The Conditions under which Gram-Charlier and Edgeworth Curves are Positive Definite and Unimodal," Biometrika, 39, 425-427.

[2] Bauwens, L., Laurent, S. and Rombouts, J. V. K. (2005) "Multivariate GARCH Models: A Survey". Journal of Applied Econometrics, forthcoming.

[3] Bollerslev, T. (1986), "Generalized Autoregressive Conditional Heteroskedasticity," Journal of Econometrics, 31, 307-327.

[4] Brown, C. A. and Robinson, D. M. (2002), "Skewness and Kurtosis Implied by Option Prices: A Correction," Journal of Financial Research, $25,279-282$.

[5] Charlier C. V. (1905), "Uber Die Darstellung Willkurlicher Funktionen," Arvik fur Mathematik Astronomi och Fysik, 9, 1-13.

[6] Christoffersen, P. and Gonçalves, S. (2003), "Estimation Risk in Financial Risk Management," Working Paper, McGill University.

[7] Corrado, C. J. and Su, T. (1996), "Skewness and Kurtosis in S\&P 500 Index Returns Implied by Option Prices," Journal of Financial Research, 19, 175-192.

[8] Draper, N. and Tierny, D. (1972), "Regions of Positive and Unimodal Series Expansion of the Edgeworth and Gram-Charlier Approximations," Biometrika, 59, 463-465. 
[9] Edgeworth, F. Y. (1896), "The Asymmetrical Probability Curve," Philosophical Magazine, 5th Series, 41.

[10] Edgeworth FY. (1907), "On the representation of statistical frequency by series," Journal of the Royal Statistical Society, series A, 80.

[11] Engel, R. F. (1982) "Autoregressive Conditional Heteroskedasticity with Estimates of the Variance of United Kingdom Inflations," Econometrica, $50,987-1007$.

[12] Gallant, R. and Nychka, D. (1987), "Seminonparametric Maximum Likelihood Estimation," Econometrica, 55, 363-390.

[13] Gallant R., Tauchen G. (1989), "Seminonparametric Estimation of Conditionally Constrained Heterogeneous Processes: Asset Pricing Applications," Econometrica, 57, 1091-1120.

[14] Harvey C. R. and Siddique A. (1999), "Autoregressive Conditional Skewness," Journal of Financial and Quantitative Analysis, 34, 465-487.

[15] Jondeau, E. and Rockinger, M. (2001), "Gram-Charlier Densities," Journal of Economic Dynamics and Control, 25, 1457-1483.

[16] Kendall, M. and Stuart, A. (1977), The Advanced Theory of Statistics, Vol. I, 4th edn., Griffin \& Co. London.

[17] León A, Rubio G, and Serna G. (2004), "Autoregressive Conditional Volatility, Skewness and Kurtosis," WP-AD 2004-13, IVIE.

[18] Mauleón I. (1997), "Instability and Long Memory in Conditional Variances," Journal de la Societe de Statistique de Paris ,134, 67-88. 
[19] Mauleón I. and Perote J. (2000), "Testing Densities with Financial Data: An empirical Comparison of the Edgeworth-Sargan Density to the Student's t," European Journal of Finance, 6, 225-239.

[20] Mauleón, I. (2003), "Financial Densities in Emerging Markets: An Application of the Multivariate ES Density," Emerging Markets Review, 4, 197-223.

[21] Niguez, T. and Perote, J. (2004), "Forecasting the Density of Asset Returns", STICERD Working Paper EM/2004/479, London School of Economics.

[22] Nabeya, S. (2001), "Approximation to the Limiting Distribution ot tand F- Statistics in Testing for Seasonal Unit Roots," Econometric Theory, 17, 711-748.

[23] Nishiyama, Y. and Robinson, P. M. (2000), "Edgeworth Expansions for Semiparametric Averaged Derivatives," Econometrica, 68, 931-980.

[24] Perote, J. (2004), "The Multivariate Edgeworth-Sargan Density," Spanish Economic Review, 6, 77-96.

[25] Perote, J. and Del Brio, E. (2003), "Measuring Value-at-Risk under the Conditional Edgeworth-Sargan Distribution," Finance Letters, 1, 23-40.

[26] Prucha, I. and Kelejian, (1984), "The Structure of Simultaneous Equation Estimation: A Generalization towards Nonnormal Disturbances," Econometrica, 52, 721-737.

[27] Rubinstein, M. (1998), "Edgeworth Binomial Trees," Journal of Derivatives, 5, 20-27. 
[28] Sargan, J. D. (1975), "Gram-Charlier Approximations Applied to t Ratios of k-Class Estimators," Econometrica, 43, 327-347.

[29] Sargan J. D. (1976) "Econometric Estimators and the Edgeworth Approximation," Econometrica, 44, 421-448.

[30] Velasco, C. and Robinson, P. M. (2001), "Edgeworth Expansions for Spectral Density Estimates and Studentized Simple Mean," Econometric Theory, 17, 497-539.

[31] Verhoeven, P. and McAleer, M. (2004), "Fat Tails in Financial Volatility Models," Mathematics and Computers Simulation, 64, 351-362. 